Available online on 15.05.2017 at http://iddtonline.info
(2011-17, publisher and licensee JDDT, This is an Open Access article which permits unrestricted
non-commercial use, provided the original work is properly cited

Mini Review

\title{
MODULATING MULTIPLE DRUG RESISTANCE VIA CO-DELIVERY OF NANOPARTICLE
}

\author{
Xialin Chen, Wenzhe Huang, Wei Xiao*
}

State Key Laboratory of New-tech for Chinese Medicine Pharmaceutical Process, Jiangsu Kanion Pharmaceutical Co. Ltd., Lianyungang 222001, China

Article Info: Received 16 March 2017; Review Completed 05 May 2017; Accepted 05 May 2017, Available online 15 May 2017 Cite this article as:

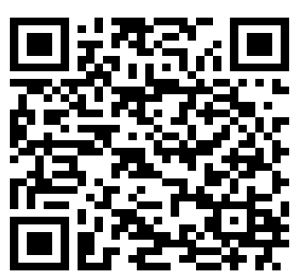

Chen X, Huang W, Xiao W, Modulating multiple drug resistance via co-delivery of nanoparticle, Journal of Drug Delivery and Therapeutics. 2017; 7(3):70-72 DOI: http://dx.doi.org/10.22270/jddt.v7i3.1424

*Address for Correspondence:

Professor Wei Xiao, State Key Laboratory of New-tech for Chinese Medicine Pharmaceutical Process, Jiangsu Kanion Pharmaceutical Co. Ltd., Lianyungang 222001, China

E-mail: kanionxw2010@126.com, Fax:+86-518-81152367

\begin{abstract}
When various mechanisms in cancer causing cells show resistance towards one or many chemotherapeutics, it hinders the efficacy of chemotherapy. This is referred as multidrug resistance. There are several factors that contribute in MDR. This research specifically focuses on modulate resistance towards multiple drugs by co-delivery of the nanoparticle. This research review has highlighted several delivery systems of nanodrug for overcoming the mechanism followed by MDR. This is done by exploiting, evading or neutralizing the pumps of drug efflux. The research essay has specifically discussed five different system categories. These categories are Mdr-1 and survivin-targeting, Docetaxel and siRNA, Paclitaxel and curcumin, Doxorubicin and paclitaxel and Doxorubicin and PSC 833. Based on the overall discussion, a brief summary is provided.
\end{abstract}

Keywords: Drug resistance, nanomedicine, co-delivery, drug delivery.

\section{INTRODUCTION}

Cancer is a major factor contributing to several deaths across the globe. There has been development of several therapeutic strategies such as biological treatments to specific pathways of a cell. ${ }^{1-3}$ However, acquired and inherent multidrug resistance (MDR) towards drugs of chemotherapy is a major challenge for effectively treating cancer. ${ }^{4,5}$ Cancer is one of the diseases that are prevailing and most impactful presently. 6,7

The aim of this essay is to modulate MDR by the use of nanoparticle via co-delivery. ${ }^{8}$ In this research essay, specific theoretical understanding will be established regarding the key concepts relevant to this research issue. ${ }^{9}$ A brief discussion on specific topics will be presented that include Mdr-1 and survivin-targeting, Docetaxel and siRNA, Paclitaxel and curcumin, Doxorubicin and paclitaxel and Doxorubicin and PSC 833 and a brief summary will be provided to conclude the essay.

\section{MDR-1 and Survivin-Targeting}

MDR is the biggest challenge faced in successfully completing chemotherapy for the treatment of cancer. ${ }^{10,11}$ There is a complicated mechanism involved with several factors affecting the same such as proteins functioning, $\mathrm{ABC}$ transporters and extrude drugs and toxins. ${ }^{12}$ Survivin is a new member of the family of apoptosis inhibitors. ${ }^{13}$ The functional role is possible in the case of apoptosis control and cell division. This is up- regulated in case of human cancers that make it a new potential target for the treatment of cancer. ${ }^{14}$ For the purpose of overcoming MDR, some compounds of the small molecule were utilized in the form of functional inhibitors like promethazine, cyclosporine A, and verapamil. However, their unknown pharmacokinetic interaction with toxicities and chemotherapeutics restricted their utilization in clinics. Additional reports were made that the encapsulation of chemotherapeutic agents within nanoparticles could end up evading the pumps of drug efflux. This further ended up increasing the concentration of the intracellular drug. 
This rarely showed the effects of anti- tumor. In addition, such increased level of concentration did not lead towards the proportionately increasing death of cell on blocking the apoptosis pathway. It was recently investigated and discovered that MDR can enable partial reversal as the gene coding expression is silenced. Their experiments on in vivo anti-tumor only ended up exhibiting the capability to reduce the growth of tumor but without decreasing the volume of the tumor.

\section{Docetaxel and siRNA}

The combination of several therapeutic approaches with various mechanisms can be a feasible option for effectively treating cancers with combined or synergistic impacts. ${ }^{15}$ Small interference of RNA was a strong technique with a feasible therapeutic option to silence genes targeted in a number of diseases. ${ }^{16}$ A number of different therapeutics based on siRNA have established for treatment of diseases effectively. ${ }^{17}$ However, there cannot be an easy crossing of cell membranes in siRNA due to the involvement of negative charge and there is a delay in downstream effects. ${ }^{18}$ This is in comparison with the conventional therapeutics based on protein or small molecule. In addition, for being successful in clinically applying siRNA, there is a requirement to be effective in delivering vehicles. This is crucial for overcoming several barriers in the cell. In addition, anti-cancer drug effect of the small molecule is fast in comparison with siRNA. This is specific in the process of intracellular uptake. Simultaneously, nanocarriers will be targeting the drugs while lowering the side effect. ${ }^{19,20}$ Therefore, the set of newly emerging therapy based on siRNA with traditional procedures of chemotherapy can be considered advantageous. Until yet, a number of promising systems for the purposes of codelivery are established on the basis of silica, liposomal and polymeric based cationic NPs. When compared, nonviral vectors based on polymer have major benefits in comparison with cationic lipids. These advantages are specific to physiological stability, large scale production, convenience, and safety. There has been an investigation of several natural and synthetic cationic polymers as carriers of siRNA or gene. These include chitosan, polylysine, and polyethyleneimine.

\section{Paclitaxel and Curcumin}

Curcumin is a polyphenol, also termed as diferuloylmethane. Its extraction is done from Curcuma longa, which is a perennial herb. ${ }^{21,22}$ This has been researched extensively considering its level of therapeutic efficacy for a number of disorders that include other inflammatory diseases such as cancer and Alzheimer's. On extensively researching curcumin, a number of its significant functions are revealed. ${ }^{23}$ It comes in interaction with a number of proteins inhibiting the performance of several kinases and controlling the activated factors of transcription. These have specific involvement in survival and proliferation of the cell. Irrespective of the effectiveness delivered by curcumin, it has limited use in the clinic because of its property of hydrophobicity and low level of in vivo bioavailability. ${ }^{24}$ However, due to the attribution of several therapeutic activities with curcumin, a solution to these issues is being searched persistently. Delivery systems of nano-sized drugs are identified as crucial for overcoming these limitations. In addition, the establishment of MDR is among the crucial factors that fail several conventional chemotherapies. Three main mechanisms are involved in which resistance to the drug is acquired by the cancer cells. These are: decreasing the intake of the drug with water solubility, intracellular modifications impacting the performance of cytotoxic drugs like shifted cycle of the cell, and increased efflux of hydrophobic drugs dependent on energy.

\section{Doxorubicin and Paclitaxel}

Chemotherapy is not considered a good option because of its undesirable negative implications on the health. ${ }^{25}$ This is supported by a low level of bioavailability along with the development and emergence of drug resistance. Several reports have stated that resistance to multiple drugs will be significantly limiting the penetration in tumor tissues by anti-cancer drugs. ${ }^{26}$ This leads towards poorly concentrated anti-cancer drugs inside the tumor which further fails the procedure of chemotherapy. ${ }^{27}$ For the purpose of overcoming these limitations, a number of drug delivery systems with effective advancement are designed by the use of various biocompatible nano-materials. A pharmaceutical strategy that is most promising is the system of polymeric nanomicelle that has been applied in a number of trials. Polymeric micelles are known for exhibiting various benefits in comparison with other delivery systems of the drug like eased production, longer circulation, and ability of targeting. In addition, specific protection will be provided to entrapped drugs of anticancer from inactivation in the media of biology. This is because of the specific structure of core-shell across the system of the polymeric micelle. In collective terms, it has been discovered that polymeric micelles are the excellent system of nano-drug delivery for chemotherapy. Even though polymeric micelles are known for significantly affecting the establishment of anti-cancer delivery systems loaded with nano drugs, there is a scope of improvement in a certain aspect. This is because 40 per cent of tumors in humans have the ability to develope resistance against agents of chemotherapy.

\section{Doxorubicin and PSC 833 (Valspodar)}

Chemotherapy can be identified as the mainstream approach for treating metastasized and localized cancers. ${ }^{28,29}$ However, the establishment of resistance from multiple drugs by cancer cells along with side effects of systemic toxic take place due to the unspecific localization of such drugs to areas without tumor is major challenges. ${ }^{30}$ There can be the occurrence of MDR by transporters of the drug efflux which are active in effluxing out drug. This results in reducing the accumulation of intracellular drugs and decreasing the level of therapeutic efficacy. The role played by P-gp within MDR has resulted in efforts for modulating the activity of P-gp. ${ }^{31}$ Several compounds known for possessing the inhibitory activities of P-gp are synthesized specifically for addressing this problem. Inhibitors of the first generation are compounds active pharmacologically which provided evidence of inhibiting P-gp even though being researched for different purposes. ${ }^{32}$ However, significant nephrotoxic and immunosuppressive effects, cardiac toxicities, poor specificity and low potencies for transporters of drug efflux end up limiting their clinical application.

\section{SUMMARY}

There is a requirement of an efficient carrier for delivering therapeutic RNA of small interference to the site of a target as a number of natural challenges have to be dealt with. ${ }^{33,34}$ 
Poly is a specific biodegradable polymer that consists of base-sensitive or acid ester bonds and amino segments. This enables PAEs for condensing the molecules of nucleic acids at physiological $\mathrm{pH}$ while releasing them across

\section{REFERENCES}

1. Chauhan V P, and Jain R K. Strategies for advancing cancer nanomedicine. Nature materials. 2013, 12: 958-962

2. Kang C, Sun Y, Wang M, and Cheng X. Nanosized camptothecin conjugates for single and combined drug delivery. European Journal of BioMedical Research. 2016, 2: 8-14

3. Kang C, Sun Y, Zhu J, Li W, Zhang A, Kuang T, et al. Delivery of Nanoparticles for Treatment of Brain Tumor. Current Drug Metabolism. 2016, 17: 745-754

4. Sun Y, Kang C, Liu F, and Song L. Delivery of Antipsychotics with Nanoparticles. Drug Development Research. 2016, 77: 393 399

5. Cheng $\mathrm{X}$. Developing organic and inorganic nanomedicine for cancer therapy. Journal of Drug Delivery and Therapeutics. 2017 7(3): 1-4 DOI: 10.22270/jddt.v7i2.1367

6. Chow E K-H, and Ho D. Cancer nanomedicine: from drug delivery to imaging. Science translational medicine. 2013, 5: 216rv214-216rv214

7. Sun Y, Kang C, Zhang A, Liu F, Hu J, Zhong X, et al. Co-delivery of dual-drugs with nanoparticle to overcome multidrug resistance. European Journal of BioMedical Research. 2016, 2: 12-18

8. $\mathrm{Xu} \mathrm{X,} \mathrm{Ho} \mathrm{W,} \mathrm{Zhang} \mathrm{X,} \mathrm{Bertrand} \mathrm{N,} \mathrm{and} \mathrm{Farokhzad} \mathrm{O.} \mathrm{Cancer}$ nanomedicine: from targeted delivery to combination therapy. Trends in molecular medicine. 2015, 21: 223-232

9. Nie S. Understanding and overcoming major barriers in cancer nanomedicine. Nanomedicine. 2010, 5: 523-528

10. Han R, Sun Y, Kang C, Sun H, and Wei W. Amphiphilic dendritic nanomicelle-mediated co-delivery of 5-fluorouracil and doxorubicin for enhanced therapeutic efficacy. Journal of Drug Targeting. 2017, 25: 140-148

11. Kang C, Qin J, Osei W, and Hu K. Regulation of protein kinase Cepsilon and its age-dependence. Biochemical and Biophysical Research Communications. 2017, 482: 1201-1206

12. Cheng $X$, and Lee $R$ J. The role of helper lipids in lipid nanoparticles (LNPs) designed for oligonucleotide delivery. $A d v$ Drug Deliv Rev. 2016, 99: 129-137; doi: 10.1016/j.addr.2016.01.022.

13. Yin Q, Shen J, Chen L, Zhang Z, Gu W, and Li Y. Overcoming multidrug resistance by co-delivery of Mdr-1 and survivintargeting RNA with reduction-responsible cationic poly ( $\beta$-amino esters). Biomaterials. 2012, 33: 6495-6506

14. Chen Y, Bian Y, Sun Y, Kang C, Yu S, Fu T, et al. Identification of 4-aminoquinoline core for the design of new cholinesterase inhibitors. PeerJ. 2016, 4: e2140

15. Liu F, Sun Y, Kang C, and Zhu H. Pegylated Drug Delivery Systems: From Design to Biomedical Applications. Nano LIFE. 2016, 6: 1642002

16. Cheng X, Liu Q, Li H, Kang C, Liu Y, Guo T, et al. Lipid Nanoparticles Loaded with an Antisense Oligonucleotide Gapmer Against Bcl-2 for Treatment of Lung Cancer. Pharm Res. 2017, 34: 310-320; doi: 10.1007/s11095-016-2063-5.

17. Zheng $\mathrm{C}$, Zheng $\mathrm{M}$, Gong $\mathrm{P}$, Deng J, Yi H, Zhang $\mathrm{P}$, et al. Polypeptide cationic micelles mediated co-delivery of docetaxel and siRNA for synergistic tumor therapy. Biomaterials. 2013, 34: 3431-3438

18. Yang Z, Xie J, Zhu J, Kang C, Chiang C, Wang X, et al. Functional exosome-mimic for delivery of siRNA to cancer: in vitro and in vivo evaluation. Journal of Controlled Release. 2016, 243: 160-171

19. Yao Z, Sun Y, and Kang C. Structure and Self-Assembly of Multicolored Naphthalene Diimides Semiconductor. Nano LIFE. 2016, 6: 1642007

20. Zhong X, Sun Y, Kang C, and Wan G. The theory of dielectrophoresis and its applications on medical and materials cytoplasm at acidic lysosomal or endosomal environment. Even though several reports are made to employ PAEs as vehicles of RNA, the efficiency of RNAi may not be as high as per the expectation set.

research. European Journal of BioMedical Research. 2017, 2: 711

21. Qiao H, Fang D, Chen J, Sun Y, Kang C, Di L, et al. Orally delivered polycurcumin responsive to bacterial reduction for targeted therapy of inflammatory bowel disease. Drug Delivery. 2017, 24: 233-242

22. Song L, Kang C, Sun Y, Huang W, Liu W, and Qian Z. Crocetin Inhibits Lipopolysaccharide-Induced Inflammatory Response in Human Umbilical Vein Endothelial Cells. Cellular Physiology and Biochemistry. 2016, 40: 443-452

23. Duan Y, Pei K, Cai H, Tu S, Cheng X, Zhang Z, et al. Strategy of integrated evaluation on treatment of traditional Chinese medicine as 'interaction of system to system'and establishment of novel fuzzy target contribution recognition with herb-pairs, a case study on Astragali Radix-Fructus Corni. Molecular and Cellular Endocrinology. 2016, 434: 219-237

24. Abouzeid A H, Patel N R, and Torchilin V P. Polyethylene glycolphosphatidylethanolamine (PEG-PE)/vitamin E micelles for codelivery of paclitaxel and curcumin to overcome multi-drug resistance in ovarian cancer. International journal of pharmaceutics. 2014, 464: 178-184

25. Sun Y, and Kang C. Self-Assembly of Peptides into Hydrogel. Journal of Organic \& Inorganic Chemistry. 2016,

26. Sun Y, Kang C, Yao Z, Liu F, and Zhou Y. Peptide-Based Ligand for Active Delivery of Liposomal Doxorubicin. Nano LIFE. 2016, 6: 1642004

27. Chen Y, Zhang W, Huang Y, Gao F, Sha X, and Fang X. Pluronicbased functional polymeric mixed micelles for co-delivery of doxorubicin and paclitaxel to multidrug resistant tumor. International journal of pharmaceutics. 2015, 488: 44-58

28. Waller A P, George M, Kalyanasundaram A, Kang C, Periasamy $\mathrm{M}, \mathrm{Hu} \mathrm{K}$, et al. GLUT12 functions as a basal and insulinindependent glucose transporter in the heart. Biochimica et Biophysica Acta (BBA)-Molecular Basis of Disease. 2013, 1832: 121-127

29. XU S-h, Chen K, CHEN M-1, ZHOU P-p, HE G-w, CUI Y-j, et al. Dynamic expression of AQP4 in early stageof ischemia/reperfusion rats and cerebral edema. Chinese Pharmacological Bulletin. 2016: 1433-1441

30. Xue X, Zhao N-Y, Yu H-T, Sun Y, Kang C, Huang Q-B, et al. Discovery of novel inhibitors disrupting HIF-1 $\alpha /$ von HippelLindau interaction through shape-based screening and cascade docking. PeerJ. 2016, 4: e2757

31. Bajelan E, Haeri A, Vali A M, Ostad S N, and Dadashzadeh S. Codelivery of doxorubicin and PSC 833 (Valspodar) by stealth nanoliposomes for efficient overcoming of multidrug resistance. Journal of Pharmacy \& Pharmaceutical Sciences. 2012, 15: 568582

32. Li H, Quan J, Zhang M, Yung B C, Cheng X, Liu Y, et al. LipidAlbumin Nanoparticles (LAN) for Therapeutic Delivery of Antisense Oligonucleotide against HIF-1alpha. Mol Pharm. 2016, 13: 2555-2562; doi: 10.1021/acs.molpharmaceut.6b00363.

33. Duan Y, Pei K, Cai H, Tu S, Zhang Z, Cheng X, et al. Bioactivity evaluation-based ultra high-performance liquid chromatography coupled with electrospray ionization tandem quadrupole-time-offlight mass spectrometry and novel distinction of multisubchemome compatibility recognition strategy with Astragali Radix-Fructus Corni herb-pair as a case study. Journal of pharmaceutical and biomedical analysis. 2016, 129: 514-534

34. Li H, Cheng X, Liu Y, Lee Y B, Kim D J, Ahn C-h, et al. Folate receptor-targeted lipid coated albumin nanoparticles (F-LCAN) for therapeutic delivery of RX-0201 (Archexin®), an antisense oligonucleotide against Akt-1. 2016. 